\title{
Design and optimization of acoustic liners with a shear grazing flow: OPAL software platform description
}

\author{
Frank Simon ${ }^{1}$, Rémi Roncen ${ }^{1}$, Pierre Vuillemin ${ }^{2}$, Patricia Klotz ${ }^{2}$, Éric Savin ${ }^{3}$, Fabien Méry ${ }^{1}$, Estelle
} Piot $^{1}$

${ }^{1}$ DMPE, ${ }^{2}$ DTIS - ONERA - Université de Toulouse

F-31055, Toulouse, France

${ }^{3}$ DTIS - ONERA - Université Paris Saclay

F-91123 Palaiseau, France

\begin{abstract}
In the context of aircraft noise reduction in varied applications where a cold or hot shear grazing flow is present (i.e., engine nacelle, combustion chamber, jet pump, landing gear), improved acoustic liner solutions are being sought. This is particularly true in the low-frequency regime, where space constraints limit the efficiency of conventional liner technology. Therefore, liner designs must take into account the dimensional and phenomenological characteristics of constituent materials, assembly specifications and industrial requirements involving multiphysical phenomena.

To perform the single/multi-objective optimization of complex meta-surface liner candidates, a software platform coined OPAL (OPtimization of Acoustic Liners) was developed. Its first goal is to allow the user to assemble a large panel of parallel/serial elementary acoustic layers along a given duct. Then, the physical properties of this liner can be optimized for a given flow and frequency range, relatively to weighted objectives: impedance target, maximum absorption coefficient or transmission loss with a total sample size and weight, etc.

The presentation focuses on the different elementary bricks and assembly of an optimization problem (from OD analytical coarse designs in order to reduce the parameter space, up to $2 D$ plan or axisymmetric high-order discontinuous Galerkin simulations of the linearized Euler equations (LEE)).
\end{abstract}

\section{INTRODUCTION}

In the context of aircraft noise reduction in varied applications where a cold or hot shear grazing flow is present (i.e., engine nacelle, combustion chamber, jet pump, landing gear), improved acoustic liner solutions are being sought.

These coatings are also subject to operational requirements in terms of aerodynamic impact, mass, thickness, temperature, static and vibration behavior, fire resistance, drainage... (see e.g., $[1,2,3]$ ).

\footnotetext{
${ }^{1}$ frank.simon@onera.fr, remi.roncen@onera.fr, fabien.mery@ onera.fr, estelle.piot@onera.fr

${ }^{2}$ pierre.vuillemin@onera.fr, patricia.klotz@onera.fr, ${ }^{3}$ eric.savin@ onera.fr
} 
In practice, the liner design implies an optimization process with acoustic single/multi-objective functions, such as impedance or transmission loss (TL), while taking into account the dimensional and phenomenological characteristics of materials (porous layer, resonator...), manufacturing, assembly specifications and industrial constraints.

However, most designs led by laboratories are based on classical architectures with reference models applied under conditions of use. Besides, locally reacting liners, such as those used in aeronautical engine nacelles, are generally "sandwich" resonators with a perforated plate linked to a honeycomb material above a rigid plate (called SDOF for Single Degree Of Freedom). Their absorption behavior can be described approximately with the Helmholtz resonator principle. To absorb in several frequency bands with one design, different types of liners with one degree of freedom can be piled up to constitute $2 \mathrm{DOF}[4,5,6]$ or $3 \mathrm{DOF}$ liners [7]. Thus, in a 2DOF liner, a septum sheet [5] (mid sheet) is used instead of the original acoustically rigid bottom plate, revealing a second cavity tuned to a dissimilar frequency. The lower cavity becomes a second degree-of-freedom, hence the name of 2DOF. The range of effective suppression can be designed to cover up to two octaves, in contrast with the characteristic single-octave coverage of typical SDOF liners [8].This, of course, implies a more complicated manufacturing process and an increased installation volume.

Nevertheless, to absorb over a wide frequency range or in a low frequency regime, it may be necessary to design non-conventional liners as, for example, variable-depth liners [9] or liners based on perforated plates connected to tubes of variable lengths that fill cavities [10]. In this case, it appears necessary to implement numerical and experimentation tools with an optimization process in order to propose configurations, such as meta-surface liners, able to satisfy required constraints.

To perform the single/multi-objective optimization of complex meta-surface liner candidates based on conventional and non-conventional architectures, a software platform coined OPAL (OPtimization of Acoustic Liners) was developed by the authors.

This paper focuses on the different elementary bricks and assembly of a problem from the acoustic liner modelling, the wave attenuation within an infinite or a finite duct solved in 1D or 2D and the optimization process.

\section{OPAL ARCHITECTURE}

The first goal of OPAL is to allow the user to assemble a large panel of parallel/serial elementary acoustic layers along a given duct. Then, the physical properties of this liner can be optimized for a given flow and frequency range, relatively to weighted objectives: impedance target, maximum absorption coefficient or transmission loss with a total sample size and weight...

The design and optimization processes can be described as follows (Figure 1) with, in input, the duct geometry, the flow characteristics and the types of parameter as constraints and targets, and, in output, the optimized liner characteristics with associated acoustical properties, a sensitivity analysis and uncertainties:

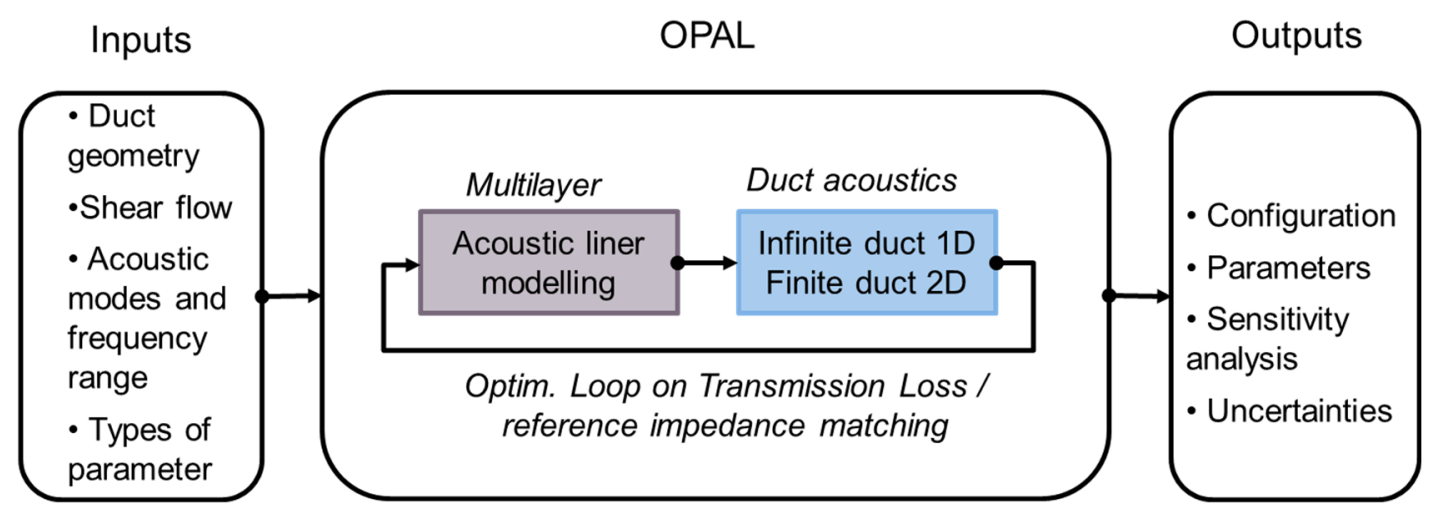

Figure 1: Design and optimization processes. 
The modelling of an acoustic liner is composed of several elements: an environment (i.e., air) in which a liner is placed, different acoustical unit materials with which to form the liner, a multilayer (ML) class to assemble sandwich materials (serial combination) and a liner class to assemble metasurfaces (parallel combinations) (Figure 2).

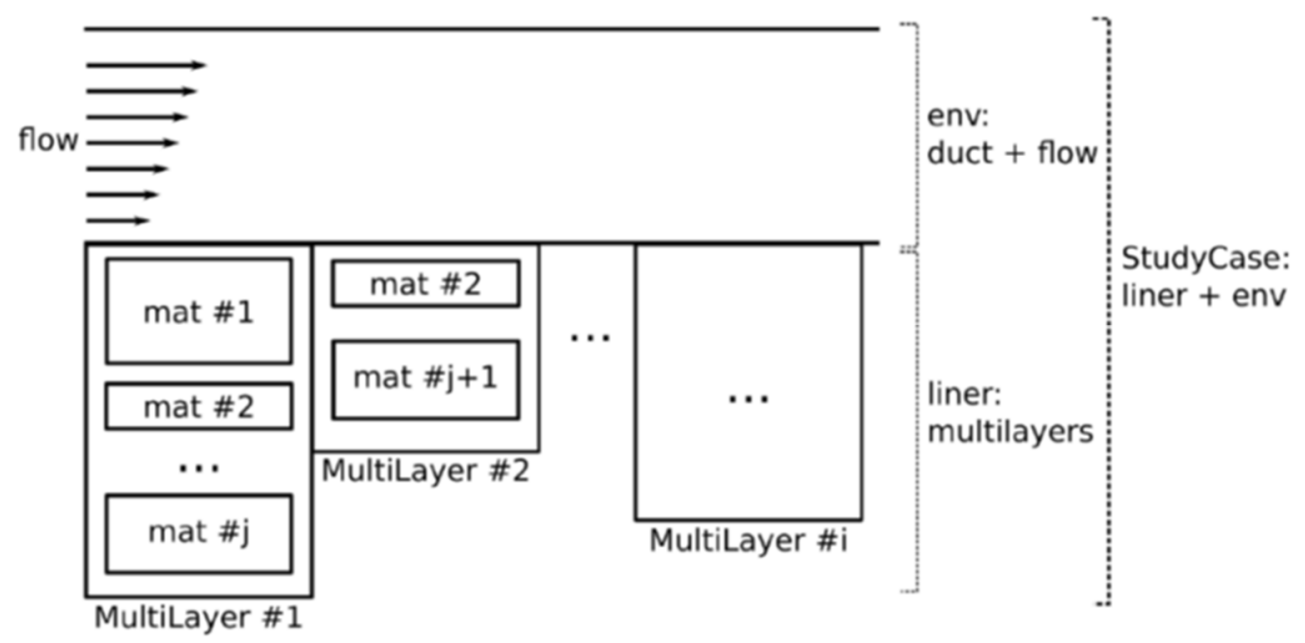

Figure 2: OPAL architecture.

\subsection{Acoustic Liner Modelling}

The multilayer classes can be made up of microperforated panel (MPP), wiremesh sheet, air or porous medium and LEONAR (Long Elastic Open Neck Acoustic Resonator [10]) (Figure 3):

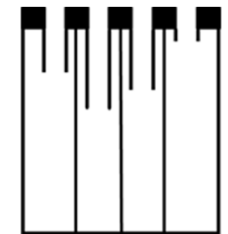

(a)

MC-LEONAR

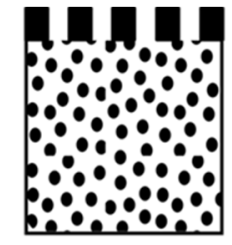

(b)

MPP-porous

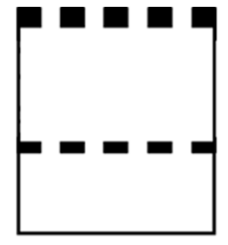

(c)

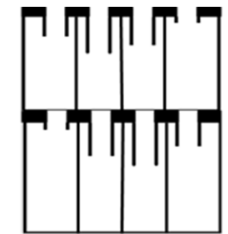

(d)

ML (MPP-air) MC (ML-LEONAR)

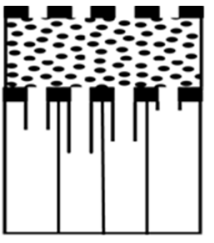

(e)

(MPP-porous) (MC-LEONAR)

(MC: multiple-cell / ML: multiple-layer / MPP: microperforated panel / LEONAR: Long Elastic Open Neck Acoustic Resonator)

Figure 3: Examples of multilayer classes.

The environment deals with the physical fluid surrounding the acoustical material of interest, the different phenomena of interest for liner studies (presence of a flow) and the acoustic content (frequencies, sound pressure level (SPL)).

Four such elementary acoustic materials can be assembled to create an acoustic liner:

- A porous material is a solid matrix filled with a fluid. A hypothesis on the rigidity of the solid matrix allows one to consider porous materials as equivalent fluids. Depending on the semi phenomenological model used to represent the pores of the material, viscous and thermal effects can be estimated with a finite set of intrinsic parameters (porosity, tortuosity, etc.).

- A microperforated plate (MPP) is, as the name indicates, a plate with micro perforations. The main difference with a classical porous material is the presence of "end effects", caused by the thinness of the plate and its low porosity. Following Atalla \& Sgard [11], a perforated plate is modelled as an equivalent fluid, with additional terms used to account for the media in contact with the perforations. Moreover, we state the modelling type of high sound pressure level (SPL) nonlinearities with Melling's model [12]. 
- Honeycomb structures are often employed in aeronautical applications, for their lightweight and structural properties. In terms of modelling, a honeycomb can be seen as a porous material with very high porosity and cylindrical straight pores of almost circular cross-shapes. In the limit where the pore radius tends to +1 (or, a bit more realistically, to $1 \mathrm{~m}$ ), the honeycomb behaves like the open ambient fluid.

- A LEONAR $[10,13]$ consists in the combination of tubes and a cavity $[14,15]$. The tubes are modeled similarly to a perforated plate of increased thickness, while the cavity is taken as a honeycomb layer of adequate radius. As such, a LEONAR cell is quite different in its implementation, when compared with the above unit materials: in itself, a LEONAR cell is already a combination of unit elements. An approximation is made, consisting in assuming that the volume occupied by the tubes inside the cavity is negligible. This is done to ensure that the classical Transfer Matrix formalism can be applied directly to LEONAR cells [16]. In practice, the upper cavity (containing the tube(s)) has an acoustic response that depends on the presence of tubes. This can be taken into account, as done in [17].

Now that we have created some materials and liners, we can evaluate their acoustical properties, as (Figure 4):

- The normalized impedance Zs

- The absorption coefficient at normal incidence.
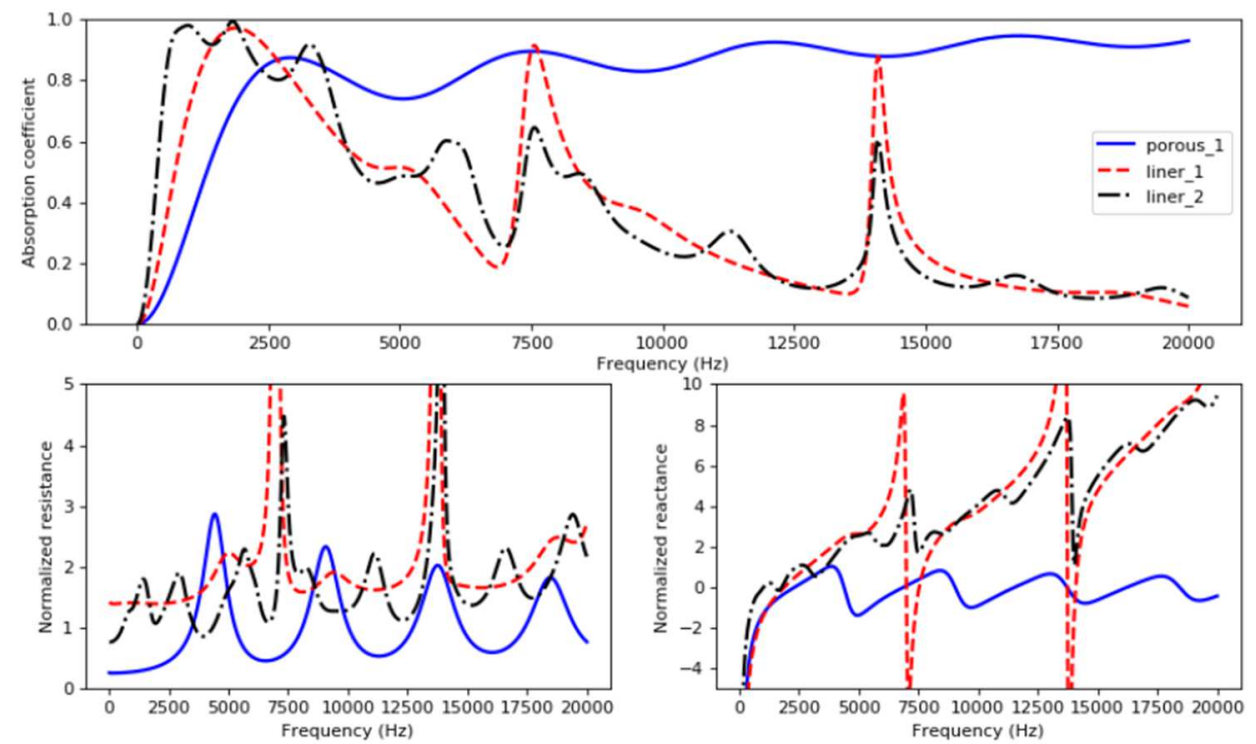

Figure 4: Normalized impedance (resistance and reactance) and absorption coefficient for a porous material and two liners at $160 \mathrm{~dB}$.

\subsection{Simulation}

Two very distinct simulation capabilities are available. The first one assumes a liner of infinite length, and solves a simplified 1D problem of wave attenuation within an infinite duct. The second problem tackles the more challenging 2D case, where impedance discontinuities as well as complex geometries can be taken into account. In both situations, both plane and axisymmetric cases can be considered.

\section{Infinite duct 1D case}

In the infinite duct 1D case, a mesh is considered in the transverse direction (perpendicular to the walls). An eigenproblem is assembled, and solved for each frequency of interest from the linearized Euler equations (LEE) discretized with a spectral collocation method (Tchebychev polynomials) [18]. 
The output of this eigen problem is a set of modes propagating in the duct, that are being attenuated due to the presence of the liner. In the case of a cylindrical duct (Figure 5), the pressure field of a given $(m, n)$ mode (azimuthal and radial) writes:

$$
p(x, r, \theta)=P_{m, n}(r) e^{-i k_{m, n} x} e^{-i m \theta}
$$

where the complex wavenumber $k_{m, n}$ is the solution of a dispersion relation.
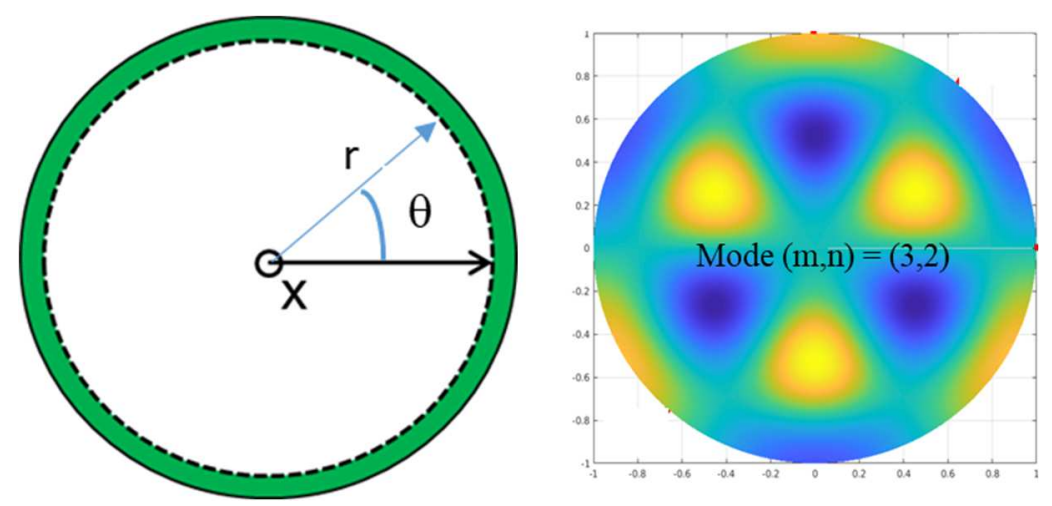

Figure 5: Cylindrical coordinates and pressure field of a mode $(3,2)$ applied to a lined cylindrical duct.

The shear flow velocity profile and its spatial derivative are an input to the problem. Each material in contact with the flow should be given a flow-nonlinearity model [20]. The layers not in contact with the flow are not considered in the calculation of the additional flow nonlinear impedance.

Regarding a given cut-on left running mode $(m, n)$, and considering an impedance value, the transmission loss coefficient $(\mathrm{dB} / \mathrm{m})$ can be directly derived from the imaginary part of $k_{m, n}$ (Figure 6):

$$
T L_{m, n} / \text { meter }=20 \log _{10}\left(\frac{p(x=1)}{p(x=0)}\right)=-20 \log _{10}(e) \operatorname{Im}\left(k_{m, n}\right) \approx-8.6859 \operatorname{Im}\left(k_{m, n}\right)
$$

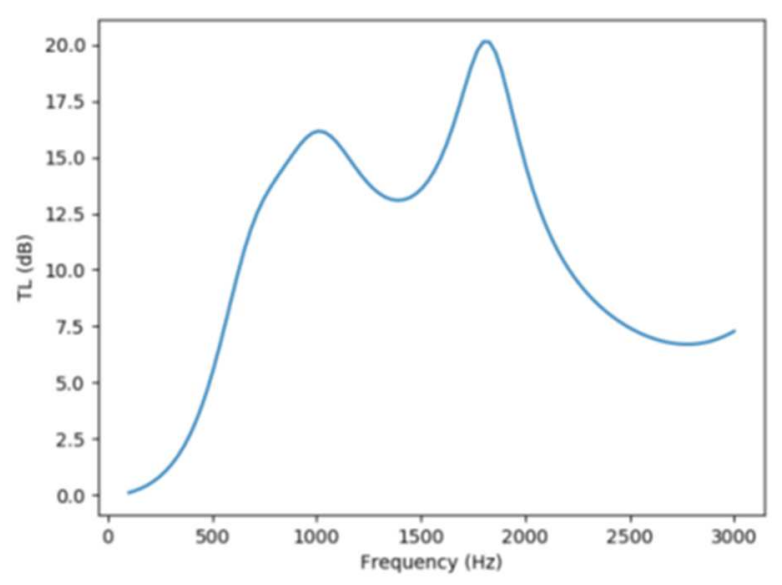

Figure 6: TL for the plane wave mode

Configuration: $5 \mathrm{~cm}$ high duct, $40 \mathrm{~cm}$ long liner, shear flow (flow model [19]) at Mach 0.3, $130 \mathrm{~dB}$, non-linearity liner model [20]. 
Finite duct 2D case

In the case where impedance discontinuities need to be taken into account, or when the geometry is complex, a 2D Discontinuous-Galerkin (DG) solver for the harmonic linearized Euler equations can be used.

We first consider a 2D plane configuration (Figure 7) with the flow profile and an environment specified in input.

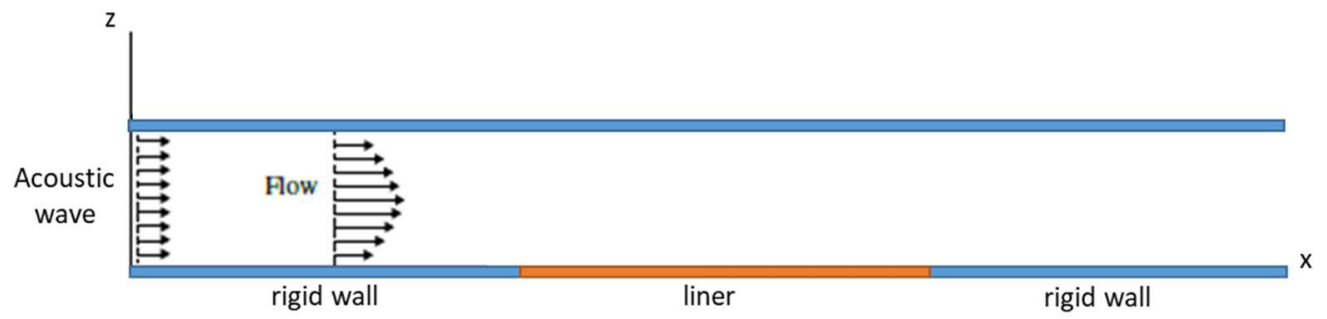

Figure 7: 2D plane configuration with a finite liner on one side and upstream/downstream rigid walls.

Typical DG orders vary between 2 and 8. Now, one has to build the LEE mass (M) and stiffness (K) matrices, in order to assemble the harmonic linear problem (Note that the stiffness matrix is separated into a main matrix $\mathrm{K}$ and a matrix related to the reflection coefficient of the liner).

The harmonic problem is then solved to plot different fields (u, v, p), such as the sound pressure level (SPL) (Figure 8 and Figure 9).

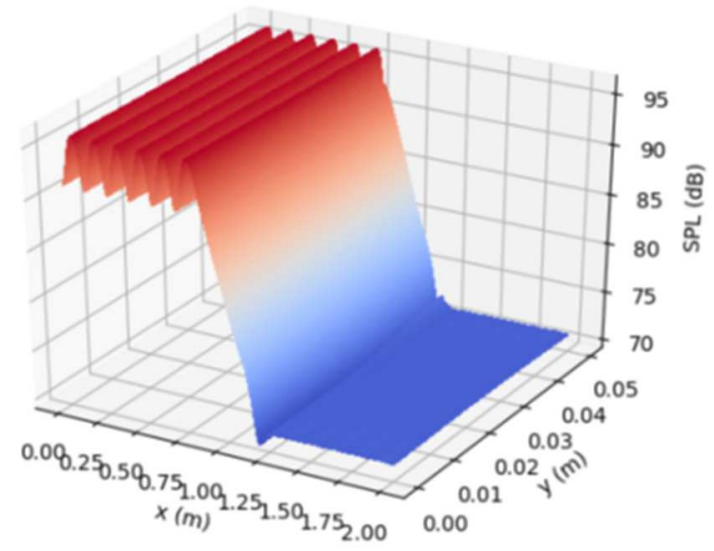

Figure 8: 3D plot of the SPL.

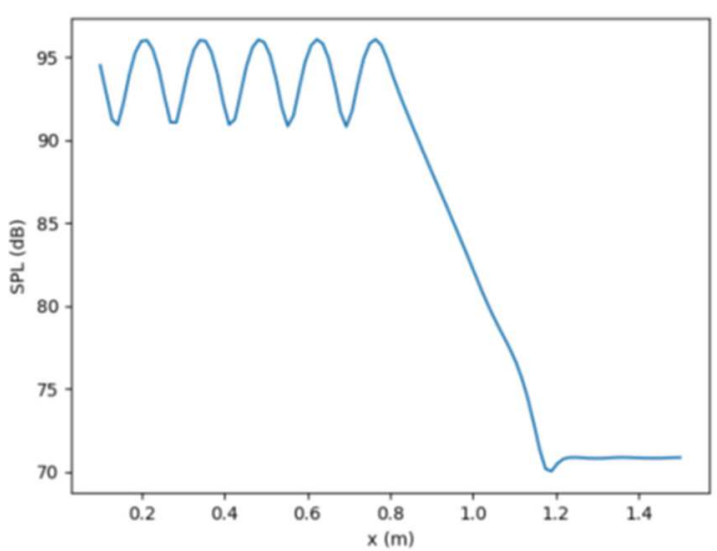

Figure 9: 1D plot of the SPL along the center line. 
For the case where an axisymmetric configuration needs to be considered, the main difference is in the building of the mass and stiffness matrices.

If interested in the wavenumbers of waves propagating in a infinite duct having a complex cross section, a 2D DG LEE for the transverse case must be used. It basically consists in building an eigenvalue problem and solving it on arbitrary geometries, with or without flow and/or impedance boundary conditions. The generalized eigen problem is solved with an Arnoldi's method [21].

\subsection{Optimization}

The optimization layer is aimed at providing the tools to transform the physical description of the liner and its environment (as illustrated by Figure 2) into a standard (non-linear) optimization problem that can then directly be fed to an optimization solver (e.g. those in Scipy). The latter can be written as:

$$
\begin{gathered}
\min _{x \in R^{n}} f(x) \\
\text { s.t. } \quad x \in\left[x_{\text {min }}, x_{\text {max }}\right] \\
A x \leq b
\end{gathered}
$$

where $x$ is the vector of optimization variables, $f$ is the objective function, A the matrix of linear constraints and $\left[\mathrm{x}_{\min }, \mathrm{x}_{\max }\right]$ the bounds.

The vector $x$ of optimization variables gathers the physical parameters associated with each material (e.g. thickness, porosity, etc.) that are set as free variables by the user. The values of the physical parameters are tied to the elements of $x$ so that any change is automatically echoed on both sides. Similarly, changing the bounds of the physical parameters directly leads to an update of $x_{\min }$ and $x_{\max }$. In this first version of OPAL, the constraints that have been identified are linear and can be divided into two groups:

- internal constraints which links various parameters within some material. For instance, for a LEONAR, the length of the tube must be smaller than the thickness of the cavity, etc.

- global constraints which are associated with the whole liner. For instance, the overall thickness of the liner is the maximum of the thickness of each multilayer.

Again, these constraints are automatically updated should the configuration be modified, e.g. by adding a new material or changing which parameters are free. The automation of the tedious and errorprone mathematical formulation of the optimization problem enables the user to focus solely on the design aspect of the problem, i.e. the definition of the objective function $\mathrm{f}$. Note that additional (potentially non-linear) constraints can easily be implemented by exploiting the routines linking $x$ with the physical parameters.

Depending on the considered application, the adequate objective function can be quite different and several variations are possible. A few interesting cases have been identified:

- optimization of the transmission loss: in the 1D case, the transmission loss $(T L)$ is one of the indicator of interest to qualify the efficiency of some acoustic liner.

In the context of liner design, several criteria may thus be derived out of it.

Indeed, assume $W$ is the set of frequencies, $T L(x, \omega)$ the transmission loss and $\beta(\omega)$ some (userdefined) weighting function,

$$
f_{T L 1}(x)=-\sum_{\omega \epsilon W} \beta(\omega) T L(x, \omega)
$$

to increase the frequency-weighted mean value of the transmission loss;

$$
f_{T L 2}(x)=\max _{\omega \in W}-\beta(\omega) T L(x, \omega)
$$

to increase the frequency-weighted minimal value of the transmission loss. 
Using the maximum instead of the sum prevents any frequency to be favored (with $\beta(\omega)$ $=1$, the optimal solution is a straight $T L$ ) but the overall problem is harder due to the additional non-smooth function added.

- reference impedance matching: to identify the physical parameters associated with some liner described by a reference impedance.

Assume $Z(x, \omega)$ denotes the impedance and that $Z_{r e f}(\omega)$ is the reference impedance, then

$$
f_{Z 1}(x)=\sum_{\omega \in W}\left|\beta(\omega)\left(Z(x, \omega)-Z_{r e f}(\omega)\right)\right|^{2}
$$

is the frequency-weighted mean square error with the reference impedance.

$$
f_{Z 2}(x)=\max _{\omega \varepsilon W}\left|\beta(\omega)\left(Z(x, \omega)-Z_{r e f}(\omega)\right)\right|
$$

is the frequency-weighted worst matching error with the reference impedance.

Similarly, using a maximum is aimed at avoiding to favor some specific frequency but leads to an harder problem.

OPAL provides a complete offer of global optimization tools for mono and multi-objective formulations with consideration of uncertainties when necessary. Examples presented are coming from evolutionary algorithms. For costly simulations, Bayesian optimization is a more adapted parsimonious technique that requires fewer simulations. Although widely expensive, robust optimization provide solutions with a performance guaranty whatever the uncertainties. Mean-Variance optimization is widely used to find solutions that make an optimal trade-off between expected performance and variability. In this approach, Chaos polynomials are associated to Bayesian optimization.

An example of robust solution on average for stochastic optimization with uncertainties on Sound Pressure Level environmental parameter is illustrated in Figure 10. The optimal robust solution is in average more relevant.

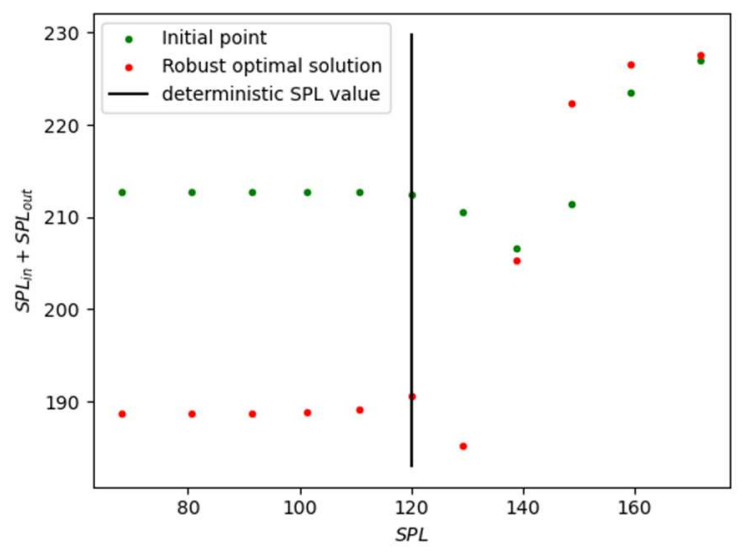

Figure 10: Comparison of robust and deterministic SPL for uncertainty on environmental SPL.

A sensitivity analysis result on a LEONAR liner with two layers, performed with a Monte Carlo on a Gaussian model is illustrated in Figure 11. The total order indices inform on how the transmission loss is affected by uncertainties on the liner parameters. Although some indices of first order are negligible, the total order indices which integrate all interactions with others parameters are all significant. 


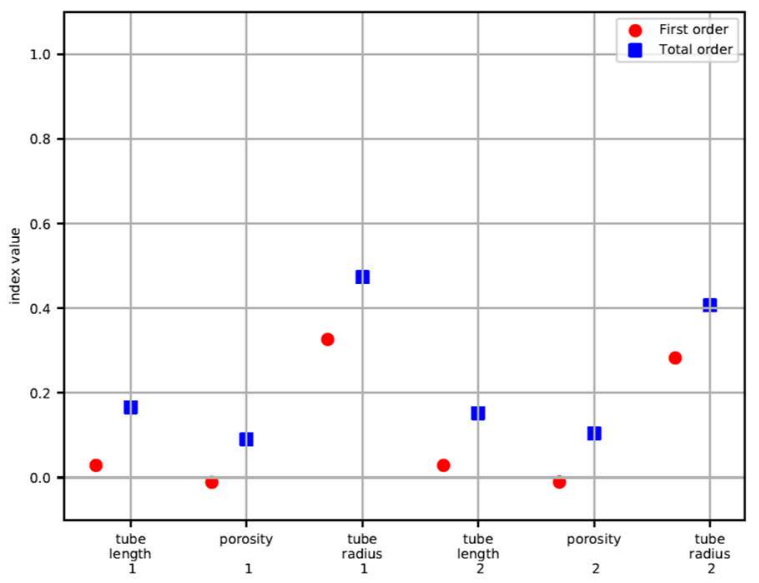

Figure 11: Sensitivity analysis on LEONAR with 2 layers.

An example of multi-objective framework coupling transmission loss and its variance is shown in Figure 12 and Figure 13 (c)). One can note that the multi-objective process tends to homogenize the transmission loss and the associated normalized impedance according to the frequency (compared to a mono-objective process applied to the transmission loss (Figure 12 and Figure 13 (a)).

It is worth noticing that the dependency in $x$ in the equation above is handled by OPAL itself and is transparent for the user. For instance, to implement the objective (6), the user simply needs (i) to ask the object that represents the liner to give its current impedance and (ii) to compute and return the mismatch with the reference impedance. See for instance the snippet of code in Figure 14 where the variables liner, env and $Z \_r e f$ are previously declared and represent a liner configuration, its environment and the reference impedance, respectively.

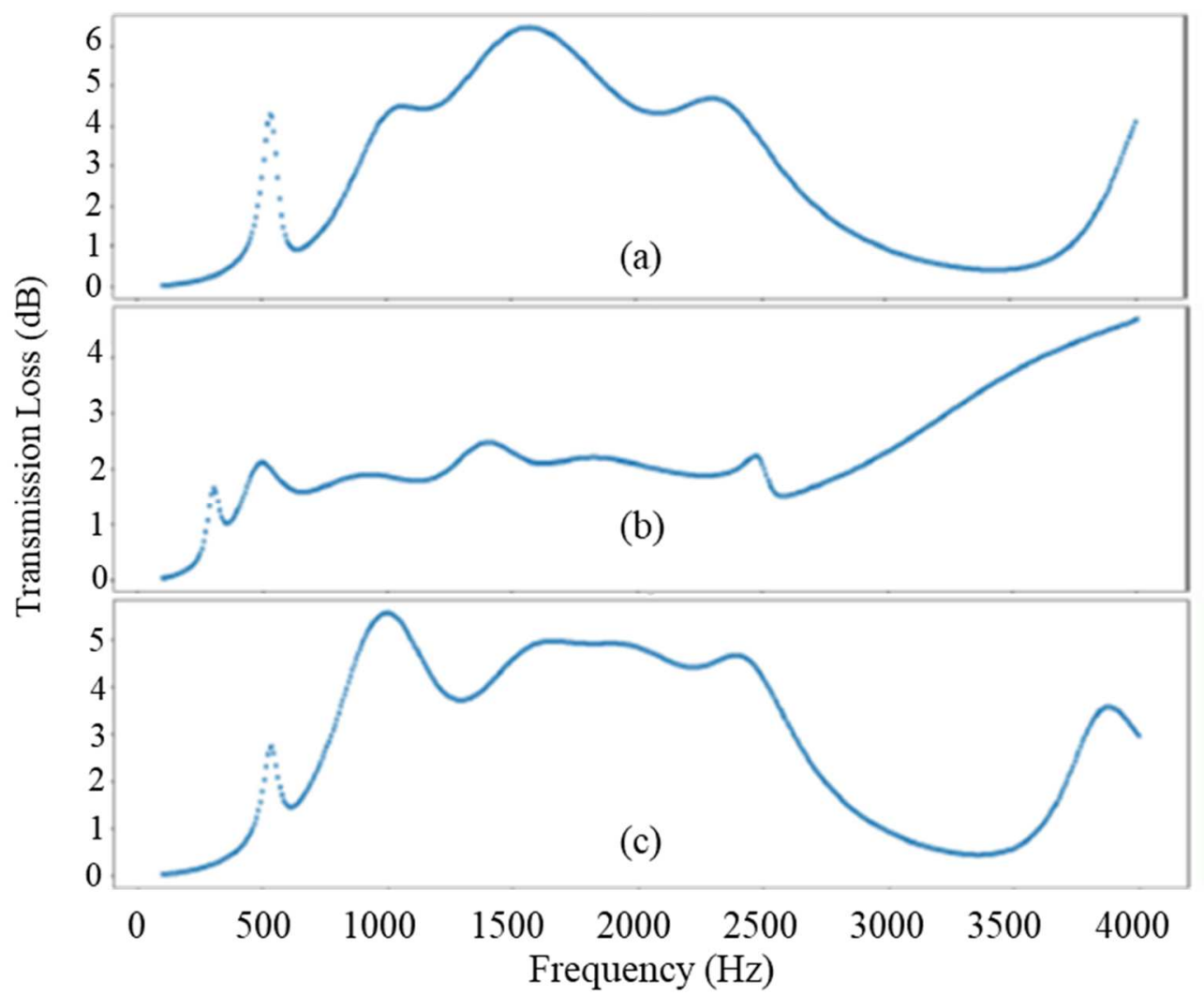

Figure 12: Plot of TL optimized vs. frequency with (a), maximization of TL, (b), minimization of variance, (c) combined criteria. 


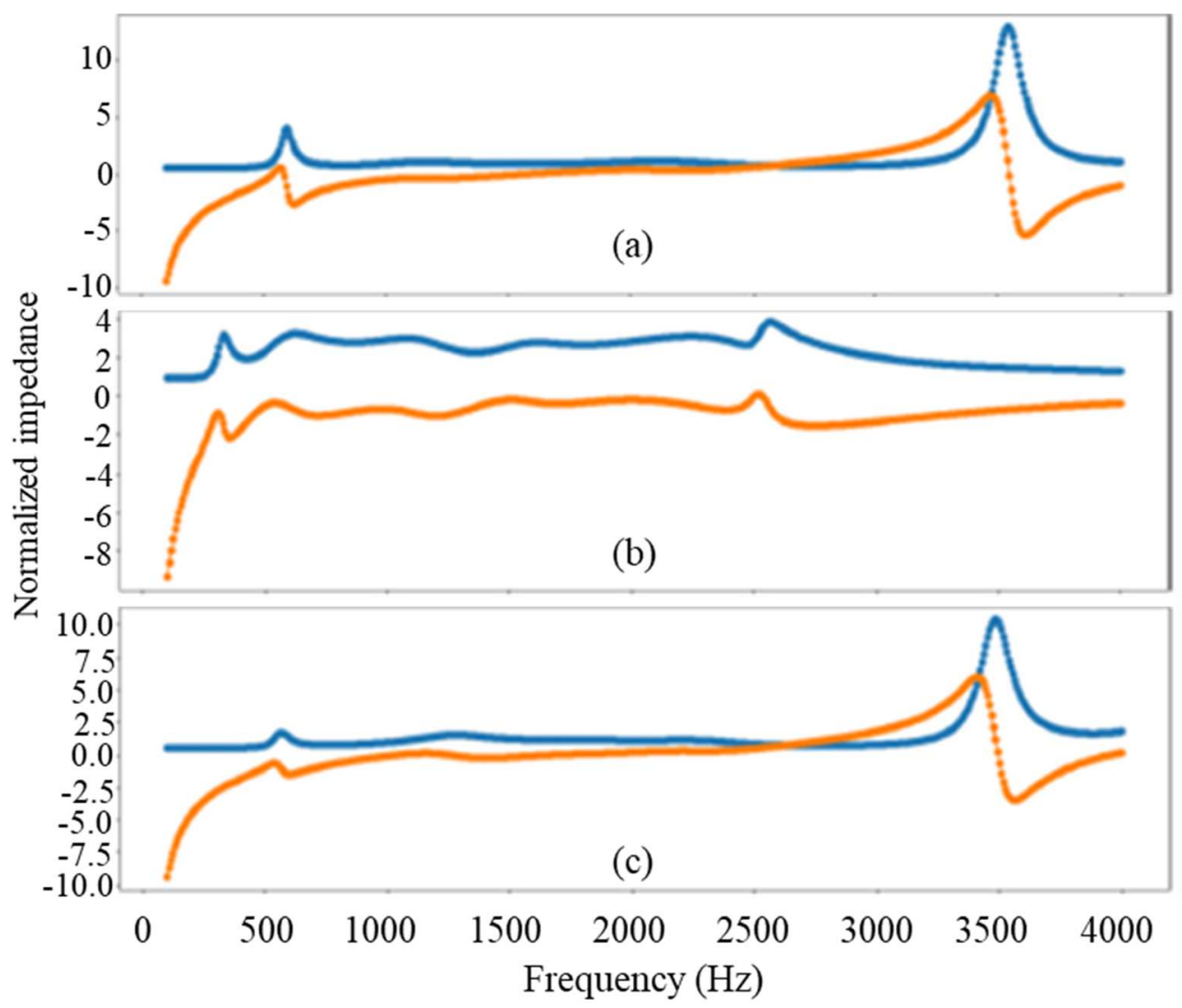

Figure 13: Plot of normalized impedance (resistance in blue, reactance in red) optimized vs. frequency with (a), maximization of TL, (b), minimization of variance, (c) combined criteria.

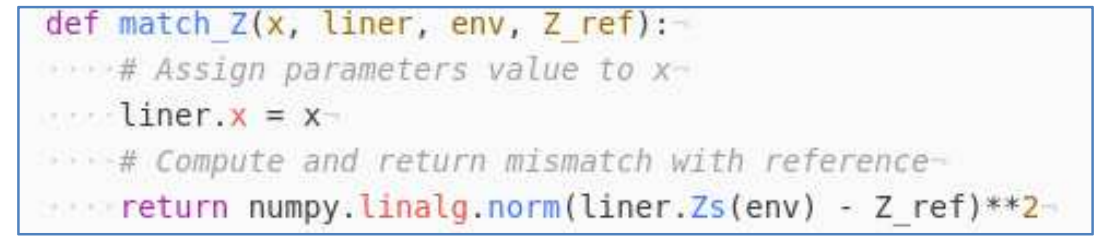

Figure 14: Possible implementation of the objective function for impedance matching without weight.

\section{CONCLUSIONS}

This paper describes a numerical approach associating aeroacoustics codes and absorbing material models in order to provide optimized liners satisfying a set of industrial specifications, as a maximum thickness and length and a target of impedance or transmission loss for a given set of acoustic modes. In [22], a numerical example is shown where an acoustic liner is optimized for a given application and experiments are conducted in ONERA's B2A aeroacoustic bench [14] with an optimized complex meta-surface assembly.

To perform realistic liners, it is necessary to introduce manufacturing or modeling uncertainties to estimate the robustness of the obtained TL.

In this case, uncertainty quantification can be deduced from a sensitivity analysis used to extract important parameters with Sobol's indices and a Bayesian calibration applied to these parameters.

\section{ACKNOWLEDGEMENTS}

We gratefully acknowledge all the ONERA contributors involved in the DOCCLA project, framework of OPAL development. 


\section{REFERENCES}

1. RTCA DO-160G. Environmental Conditions and test Procedures for Airborne Equipment.

2. Howerton, B. M. \& Jones, M. G. A Conventional Liner Acoustic/Drag Interaction Benchmark Database. AIAA Paper, 2017-4190, June 2017.

3. Lafont, V., Méry F., Reulet, Ph. \& Simon, F. Surface temperature measurement of acoustic liners in the presence of grazing flow and thermal gradient. Experiments in Fluids, 62-82 (2021).

4. Hersh, A.S., Walker, B. \& Celano, J.W. Effect Of Grazing Flow and SPL On Impedance Of 2Dof Resonators. Proceedings of the 8th AIAA/CEAS Aeroacoustics Conference And Exhibit, Breckenridge, USA, June 2002.

5. Syed, Asif A. Acoustic manufacture, General Electric Company. US Patent 6,203,656 B1 S Mar. 20 (2001).

6. Ahuja, K. K., Cataldi, P. \& Gaeta, Jr., R. J. Sound absorption of a 2DOF resonant liner with negative bias flow. CR 2000-210637, NASA (2000).

7. Julliard, J., Antoine, H., Riou, G. \& Pillut-Lesavre, S. Development of a three degree of freedom liner. Proceedings of the 7th AIAA/CEAS Aeroacoustics Conference and Exhibit, Maastricht, Netherlands, May 2001.

8. Motsinger, R. \& Kraft, R. Design and performance of duct acoustic treatment. NASA . Pub. 2 (1258), 165-206 (1991).

9. Jones, M. G., Howerton, B. M. \& Ayle, E. Evaluation of Parallel-Element, Variable Impedance, Broadband Acoustic Liner Concepts. AIAA Paper, 2012-2194, June 2012.

10. Simon, F. Garniture Surfacique pour Absorption Acoustique. INPI Patent 3,065,570, May 2019.

11 Atalla, N. \& Sgard, F. Modeling of perforated plates and screens using rigid frame porous models. J. Sound Vib., 303, 195-208 (2007).

12. Melling, T. H. The Acoustic Impedance of Perforates at Medium and High Sound Pressure Le vels. J. Sound Vib., 29(1), 1-65 (1973).

13. Simon, F. Low frequency sound absorption of resonators with flexible tubes. Proceedings of the $21^{\text {st }}$ International Congress of Acoustics, Montréal, Canada, June 2013.

14. Simon, F. Long Elastic Open Neck Acoustic Resonator for Low Frequency Absorption. J. Sound Vib., 421, 1-16 (2018).

15. Simon, F. \& Sebbane, D. Compact 2DOF Liner Based On A Longe Elastic Open Neck Acoustic Resonator For Low Frequency Absorption. Noise Control Engineering Journal, 69(1), ISSN 0736-2501, 1-17 (2021).

16. Simon, F., Mery, F., Roncen, R., Sebbane, D. \& Piot, E. Inlet Liner Design for aircraft air conditioning system. Proceedings of INTERNOISE 2019, Madrid, Spain, June 2019.

17. Li, D., Chang, D., \& Liu, B. Enhancing the low frequency sound absorption of a perforated panel by parallel-arranged extended tubes. Applied Acoustics, 102, 126-132 (2016).

18. Boyer, G., Brazier, J.P., \& Piot, E. Theoretical investigation of hydrodynamic surface mode in a lined duct with sheared flow and comparison with experiment. J. Sound Vib., 330, 1793-1809 (2011).

19. Rienstra, S.W. Fundamentals of duct acoustics. Von Karman Institute Lecture Notes, 2015.

20. Guess, A. W., Calculation of perforated plate liner parameters from specified acoustic resistance and reactance. J. Sound Vib., 40(1), 119-137 (1975).

21. Roncen, R., Piot, E., Mery, F., Simon, F., Jones, M.G. \& Nark, D.M. Wavenumber-based Impedance Eduction with a Shear Grazing Flow. AIAA Journal, 58(7), 3040-3050 (2020).

22. Roncen, R., Vuillemin, P., Klotz, P., Simon, F., Mery, F., Sebbane, D. \& Piot, E. Design and optimization of acoustic liners with a shear grazing flow: OPAL software applications. Proceedings of INTERNOISE 2021, Washington, USA, August 2021. 